ÉGYPTE monde arabe

\section{Égypte/Monde arabe}

$34 \mid 1998$

Droits d'Égypte : histoire et sociologie

\title{
Débat autour de... Hervé Bleuchot, Les cultures contre l'homme?
}

\section{Baudouin Dupret et Hervé Bleuchot}

\section{(2) OpenEdition \\ 1 Journals}

Édition électronique

URL : https://journals.openedition.org/ema/1540

DOI : 10.4000/ema. 1540

ISSN : 2090-7273

\section{Éditeur}

CEDEJ - Centre d'études et de documentation économiques juridiques et sociales

\section{Édition imprimée}

Date de publication : 31 décembre 1998

Pagination : 265-271

ISSN : 1110-5097

\section{Référence électronique}

Baudouin Dupret et Hervé Bleuchot, « Débat autour de... Hervé Bleuchot, Les cultures contre

I'homme? », Égypte/Monde arabe [En ligne], 34 | 1998, mis en ligne le 08 juillet 2008, consulté le 07

juillet 2022. URL : http://journals.openedition.org/ema/1540; DOI : https://doi.org/10.4000/ema.1540

Ce document a été généré automatiquement le 7 juillet 2022.

Tous droits réservés 


\title{
Débat autour de... Hervé Bleuchot, Les cultures contre l'homme?
}

\author{
Baudouin Dupret et Hervé Bleuchot
}

\section{RÉFÉRENCE}

Hervé Bleuchot, Les cultures contre l'homme? Essai d'anthropologie historique du droit pénal soudanais, Aix-en-Provence, Presses universitaires d'Aix-en-Provence, 1994

1 La structure du livre de Hervé Bleuchot est classique : il s'agit de dérouler l'histoire des dispositions normatives d'ordre pénal que les différentes régions et peuplades de ce qui constitue aujourd'hui le Soudan ont connues et pratiquées. À cette fin, il distingue non moins classiquement deux périodes, l'une traditionnelle, l'autre moderne. Le lecteur bénéficie dès lors d'une synthèse relativement exhaustive de la littérature consacrée au droit pénal soudanais et à son histoire. En termes anthropologiques et, plus particulièrement, en termes d'anthropologie du droit, l'ouvrage s'attaque aux notions fondatrices de la discipline, culture et pluralisme juridique essentiellement, qu'il tente de confronter à la problématique plus philosophique des droits de l'homme et des peuples.

Chronologiquement, Les cultures contre l'homme balaye une période très large s'étendant de la constitution du droit musulman à la fin de la présence anglaise, avec un épilogue aboutissant au dernier régime parlementaire. On peut se demander quel est le statut anthropologique de la section d'introduction au droit musulman (ch. 3, section 1). À tort peut-être, je me suis personnellement engagé dans la déconstruction de ce type de relation directe et mécanique entre la théorie juridique dite classique et les pratiques temporellement et localement situées.

Ainsi, pour des raisons évidentes, le XIX ${ }^{\mathrm{e}}$ siècle constitue le véritable point de départ de l'étude historique menée par $\mathrm{H}$. Bleuchot. On ne peut qu'insister sur son excellente information bibliographique, même si le spectre des situations considérées semble bien large (Bertî, Azandé, Didinga, Dinka, Nuer, Nuba, Dar Fung et Darfur, Luo, etc.). Par 
ailleurs, le traitement de sources anthropologiques de seconde main aurait sans doute supposé de conserver un regard plus critique sur la réalité qu'elles sont censées rapporter. L'histoire du droit pénal soudanais, dans le cadre du système traditionnel, couvre le Darfur et le Dar Fung, l'époque égyptienne, l'époque mahdiste et les zones païennes. La façon de le traiter n'est pas sans rappeler la structure des précis de droit. Les procédures sont distinguées des infractions, elles-mêmes réparties en infractions à compensation et infractions sans compensation. La systématique gagne à ce type de traitement ce qu'une anthropologie critique du droit y perd. Le sentiment demeure, en effet, que les normes et pratiques sont évaluées à l'aune d'un paradigme, celui du droit occidental, là où on aurait été en droit d'attendre une réflexion anthropologique diluant le droit dans le phénomène plus large de la norme. On peut à cet égard relever tout ce qu'une notion telle que la coutume doit à l'ethnocentrisme juridique et anthropologique, particulièrement quand celui-ci prend pour voix les hérauts de la codification du droit coutumier. Cette critique peut être adressée, de manière générale, à la théorie du pluralisme juridique qui, pour faire valoir le droit à l'expression d'autres formes juridiques que celles consacrées par l'État, prend pour étalon de juridicité la loi de ce même État.

4 La deuxième partie du livre s'attache à l'étude de ce que $\mathrm{H}$. Bleuchot appelle le "système moderne ». Comme dans la première partie, l'auteur fait d'abord l'historique de la période, avant de s'engager dans l'étude spécifique du droit pénal où il distingue également procédures et infractions en les mettant en perspective du droit coutumier et de sa pratique réminiscente. La qualité des sources et leur traitement sont impressionnants. L'auteur semble avoir réuni tout ce que la littérature touchant au droit pénal soudanais - et pas seulement - pouvait offrir. Le lecteur trouve ainsi une excellente information sur les motivations ayant conduit au triple refus des droits anglais, musulman et égypto-français ainsi qu'à l'adoption d'un système pénal spécifique (ch. 5 , sections 1 et 2 ). La réaction du colonisateur anglais à la "coutume » locale et ses tentatives d'élaboration d'un droit nouveau qui tienne compte des pratiques locales et transfère l'administration de la justice aux "indigènes", conformément aux politiques de la Native Policy ou de la Local Administration, font également l'objet d'un compte rendu détaillé. Le problème viendrait plutôt davantage d'une tendance à considérer les textes de loi et de jurisprudence comme constitutifs, en tant que tels, d'une réalité juridique que l'auteur veut apprécier dans sa dimension anthropologique. La critique, qui peut toujours être parée par l'invocation des sources disponibles, ramène à cette objection déjà formulée mais encore plus fondamentale à ce stade du livre l'anthropologie du droit, en tant qu'anthropologie plus générale de la norme, est une étude des pratiques fondant un droit, ses textes constitutifs et leur interprétation et non, à l'inverse, l'inventaire des dispositions applicables et des décisions de justice censées déterminer la pratique des individus et des groupes sociaux. L'étude des textes législatifs et jurisprudentiels, pour incontournable qu'elle soit - elle constitue même légitimement un objet d'étude anthropologique -, ne peut se limiter à une énumération, laissant entendre soit que ces textes sont compatibles les uns avec les autres, soit qu'ils sont contradictoires, soit encore qu'ils englobent à eux seuls le droit et ses pratiques. La relation de la norme au texte énonçant la norme n'est ni nécessaire ni suffisante, mais bien contingente et aléatoire.

5 Le livre s'achève sur un épilogue consacré à la période postérieure à l'indépendance scandée par des épisodes parlementaires et militaires. L'auteur tente d'y expliquer l'émergence progressive d'une législation pénale fondée sur des principes d'inspiration 
islamique. L'organisation de son propos reste identique d'abord historique, ensuite juridique. Quatre pages sont enfin consacrées à avancer les éléments d'une discussion. On en retiendra une affirmation à laquelle, personnellement, je ne puis que souscrire : le point de vue de l'anthropologue n'a pas à dire ce qu'est l'islam authentique. En d'autres termes et, comme le dit Clifford Geertz, aussi hérétique que puisse paraître le propos pour le croyant, l'anthropologie considère comme religion ce que les hommes affirment tel. Le point de référence transcendantal, lui, reste complètement étranger et donc, aussi, l'appréciation de l'orthodoxie. Le Code pénal soudanais, qui prétend traduire juridiquement les impératifs de la shari'a, ne peut être évalué dans sa conformité plus ou moins grande avec l'idéal d'une «Grande Tradition » quelconque. Il s'agit, tout au contraire, de le considérer de manière située, c'est-à-dire dans le contexte social et politique du Soudan des vingt-cinq dernières années. C'est ici que se situe toute la différence entre l'énoncé des dispositions d'un texte et l'analyse anthropologique des conditions de sa rédaction, de son interprétation et de son application, voire de son instrumentalisation. De ce point de vue, je ne crois pas conviction qui reste toujours toute personnelle - que le droit islamique et son histoire aient un sens ou une logique, que les musulmans aient une tendance à choisir des solutions d'un type particulier ou encore qu'ils aient à collectivement se rendre compte de quelque chose. Je prétends, pour ma part, que le droit appartient à ces « moyens du bord» dont dispose tout individu, dans un contexte social donné. Les règles de droit sont en ce sens abstraites de leur signification d'origine et ne constituent plus que des formes, des traces de l'histoire, que l'interaction sociale conduit à ponctuellement et localement investir.

Assez naturellement, mes dernières remarques porteront sur le cadre conceptuel général de l'ouvrage de $\mathrm{H}$. Bleuchot. Il convient d'abord de saluer le mérite de sa tentative d'approche anthropologique des droits de l'homme. Comme le fait remarquer Norbert Rouland dans sa préface, nombreuses doivent être les personnes qui considéreront qu'il y avait une certaine hardiesse à mêler anthropologie et philosophie politique. La difficulté ne vient pas, me semble-t-il, d'une volonté d'examiner les droits de l'homme d'un point de vue anthropologique, mais bien de la volonté de jauger des pratiques que l'on prétend examiner avec le regard anthropologique au prorata de leur conformité à la philosophie des droits de l'homme. Établir pareil critère ne revient-il pas à ce que Bleuchot pointe très justement dans les propos de son interlocuteur de la Cour suprême (p. 427-428) rétablir un point de vue normatif. Une autre remarque porte sur l'utilisation faite par $\mathrm{H}$. Bleuchot de la théorie du pluralisme juridique. La pertinence du recours à cette théorie semble tout à fait évidente. On se demande par contre pourquoi l'auteur n'ajoute pas à son analyse les travaux de John Griffiths, de Gordon Woodman, des frères von Benda-Beckman ou de Sally Engle Merry, dont on peut penser qu'ils ont largement contribué à élargir le débat. L'utilité de la théorie de Massaji Chiba semble pour sa part avérée, même si le concept d'acculturation et, partant, celui de culture juridique suscitent quelques interrogations et réticences. Je ne m'étendrai par ailleurs pas sur la faiblesse de l'opposition entre systèmes traditionnel et moderne. Qu'il suffise de dire qu'une pareille dichotomie ne semble pas très utile d'un point de vue heuristique, mais que, ontrairement à ce que l'auteur affirme dans un article ultérieur, elle ne détermine le travail que dans son organisation très générale. Enfin, ce n'est pas faire injure au livre de Hervé Bleuchot que de dire qu'il est loin d'épuiser les possibilités de l'anthropologie du droit dans les contextes soudanais. Des études prenant appui sur l'observation des lieux de production juridique pourraient 
ainsi être menées qui, tout en ayant à l'esprit l'évolution historique que le livre nous décrit, rendraient à la norme de droit son véritable statut, celui de produit des pratiques d'énonciation, d'interprétation, d'application, etc.

Les lignes qui suivent ont été rédigées par Hervé Bleuchot lui-même, suite à sa lecture du compte-rendu de B. Dupret. Nous publions ici les réflexions suscitées par cet échange vivant.

On est obligé, quand on écrit une thèse, de poser une problématique, de fixer un cadre théorique, de donner ses hypothèses de départ et sa méthode de travail. Sans cela, on court au hasard après plusieurs problèmes, on a des préconceptions mal élucidées, on ne sait plus comment procéder, ou pire, on fait de la propagande. Il est certain que la garantie d'objectivité n'est donnée que par rapport à ce cadre de travail. Il est certain aussi qu'elle n'est jamais parfaite. Mais la partialité consiste à cacher ces préconceptions. Toute recherche qui fait l'économie de cet éclaircissement est soupçonnable.

Quand on a choisi de confronter les cultures et les droits de l'homme, on pose comme point de départ que l'on va juger les cultures à l'aune des droits de l'homme. C'est clair. Il n'y a pas de trahison. Tout ce qu'on peut me reprocher c'est d'avoir pris ce sujet. Mais c'est le problème que se posent tous nos contemporains à propos de l'islam et il faut y répondre par une démarche la plus rigoureuse possible. C'est ce que j'ai tenté de faire sur un cas précis, le Soudan, pour éviter de me noyer - comme tant d'autres - dans un débat sans limites (cf. p. 93, essentiel), mais permettant d'inclure le dépassement que je souhaite il faut placer l'analyse du droit musulman dans des cadres plus larges, incluant des droits africains et européens, etc. Tout le chapitre 2 d'ailleurs exprime ma démarche.

Dire comme l'écrit B. Dupret «les normes et pratiques sont évaluées à l'aune d'un paradigme, celui du droit occidental » et «établir pareil critère ne revient-il pas à ce que Bleuchot pointe très justement dans les propos de son interlocuteur de la Cour suprême : rétablir un point de vue normatif» me semble un peu rapide. Les deux démarches ne sont pas à mettre sur le même plan. Je ne reproche pas à mon interlocuteur soudanais d'avoir une norme, mais de ne pas l'expliciter, en sorte qu'il suppose le problème résolu avant de l'examiner... Surtout, je lui reproche, sur le plan théologique, de donner une image de Dieu repoussante. Je parle aussi de l'insuffisante maitrise de la mémoire, etc. (p. 429). Je ne dénonce chez lui que les préconceptions mal élucidées, idéologiques. Vraiment, je ne pense pas que les deux démarches soient identiques.

Lors de la soutenance, on a souligné que le mot qui revenait le plus souvent chez moi était « humanisme ", car les droits de l'homme tels que je les conçois (cf. p. 66-69), c'est avant tout ce sens moral implicite. Mais alors dira-t-on, réintroduire la morale, c'est réintroduire la norme! Cessons de nous voiler la face... Pourquoi écrivons-nous ? Pourquoi étudier le droit musulman, les sociétés arabes, l'islamisme, etc.? Est-ce seulement pour décrire? N'est-ce pas parce que nous avons quelque chose à dire sur les débats actuels? L'anthropologie n'a-t-elle pas une visée morale? Le pluralisme juridique ne cherche-t-il pas à proposer une autre vision du droit et de la société plus large qui résoudrait bien des conflits, qui serait plus juste envers les minorités, etc. ? (cf. p. 78-79). Que dire alors de la «déconstruction» du droit? Notre démarche de 
savants n'est-elle pas la même pour tous : élargir les débats, changer les perspectives, prendre du recul pour dépasser les problèmes? «Dépasser » et non ignorer. Je suis persuadé que ceux qui nient ce que je viens de dire essaient de brouiller les cartes (souvent par l'accumulation d'abstractions) en sorte qu'on ne voit pas où sont leurs normes, et où ils se situent. Ma norme, c'est l'humanisme, et contrairement à beaucoup de gens, j'ai tout fait pour qu'on la voie (cf. note 51, p. 79 ou p. 205). J'ai été honnête, sans plus.

11 Au-delà de ma thèse, le débat concerne l'anthropologie juridique. B. Dupret écrit : « Le problème viendrait plutôt davantage d'une tendance à considérer les textes de loi et de jurisprudence comme constitutifs, en tant que tels, d'une réalité juridique que l'auteur veut apprécier dans sa dimension anthropologique ». S’il avait écrit « constituant une », je me serais reconnu dans cette tendance. Le droit en effet est pour moi un produit social qu'il faut expliquer. Ma démarche est durkheimienne si l'on veut, mais elle est constitutive de la sociologie: il s'agit d'expliquer un phénomène par d'autres phénomènes. Mais à l'affirmation que le droit est constituant de la société, je ne peux souscrire que si l'on ajoute que la société est constituante du droit, donc qu'il y a une dialectique droit/société.

B. Dupret dit aussi, p. 2: «l'anthropologie du droit, en tant qu'anthropologie plus générale de la norme, est une étude des pratiques fondant un droit, ses textes constitutifs et leur interprétation et non, à l'inverse, l'inventaire des dispositions applicables et des décisions de justice censées déterminer la pratique des individus et des groupes sociaux ». Il y a d'autres formules du même genre dans ce texte. Pour moi, cette formule est réductrice de l'anthropologie juridique qui ne serait que l'étude des pratiques fondant un droit. Et celle des pratiques engendrées par le droit? Il faudrait donc exclure du champ de l'anthropologie juridique la double vente et autres ruses destinées à tourner l'interdiction de l'usure? Il faudrait ne pas tenir compte des pratiques destinées à tourner l'interdiction de l'adoption? Je ne pense pas que B. Dupret aille jusque-là... Il y a toujours un "feed-back». Et dans cette querelle, qui est fort ancienne, sur le "feed-back", on ne cherche qu'à résoudre le problème de l'antériorité de la poule et de l'œuf. Si la société engendre le droit, d'accord. Mais il n'y a pas de société sans droit, donc quel est le droit qui a préexisté à la société qui engendre et qui l'a influencée, et quelle est la société qui a formé ce droit antérieur, etc. ?

13 À mon sens, il ne faut pas réduire l'anthropologie juridique à une seule démarche. Elle a deux démarches, de la société au droit, du droit à la société. La documentation peut imposer de privilégier tantôt une démarche, tantôt l'autre, le mieux étant de les associer et de saisir la dialectique entre les deux termes. C'est ce que j'ai essayé de faire, sans jamais privilégier vraiment un aspect (cf. p. 206, dernières lignes) : les parties historiques correspondent à la démarche société $\rightarrow$ droit, les parties systématiques à la démarche droit $\rightarrow$ société. Chaque partie, avec son chapitre historique et son chapitre systématique, fait clairement référence à une sorte de structuralisme génétique (cf. Piaget, par exemple). Mais les contraintes documentaires faussent souvent les perspectives, et je m'en suis méfié constamment, mettant souvent en garde le lecteur (cf. p. 112-113).

14 Je crois aussi qu'il y a un autre problème qui nous divise, et qu'il faut bien voir. C'est le rôle que j'attribue à l'histoire. J'ai longtemps travaillé avec des sociologues et des politologues. J'ai fini par les quitter parce que je leur reproche le plus souvent de 
privilégier les abstractions aux faits. Rien ne vaut un fait. Qu'il y ait eu une expansion commerciale au XVIII siècle au Soudan me ravit, car je tiens le principe explicatif de l'expansion du droit musulman à cette époque et du refoulement du droit païen des monarques du Sennar. Je réduis la sociologie à l'histoire, peut-être bien. Mais en tout cas, loin de moi l'idée que par la suite le droit musulman aille favoriser, voire provoquer diverses transformations sociales... Dialectique, histoire, bref, surtout G. Gurvitch, Balandier. Oui, « acculer la sociologie à l'histoire », comme on l'a reproché à ces maîtres, fait bien partie de mon credo.

Sur le plan théorique, il est certain que je suis resté fidèle à mes vieux maitres, délaissant nombre de théoriciens actuels, qui ne m'ont pas convaincu pour ce que j'en ai lu. B. Dupret a raison, peut-être, de me reprocher quelques silences dans ce domaine. En tout cas je le remercie d'avoir lu sérieusement mon travail. Car je suis fort déçu jusqu'ici de la paresse des analystes à qui j'ai envoyé mon livre : quand ils se décident à faire quelque chose (plus de la moitié ne font rien malgré les promesses I), ils ne lisent pas, ils ne vont pas au fond des choses et n'amorcent pas des discussions de fond, les seules qui font avancer les sciences humaines, si difficiles. Au-delà de nos deux personnes, j'espère que le débat sur la nature de l'anthropologie juridique sera poursuivi, approfondi, dépassé...

INDEX

Mots-clés : Soudan

\section{AUTEURS}

\section{BAUDOUIN DUPRET}

CNRS/Cedej

Page sur le site du Cedej

HERVÉ BLEUCHOT

CNRS/IREMAM 